\title{
Hemorragia digestiva alta como manifestação atípica de síndrome de Mirizzi
}

\author{
Upper gastrointestinal bleeding as an atypical manifestation of Mirizzi syndrome \\ Maria Carolina Galli Mortati ${ }^{1}$, Caio Barbosa Kaku ${ }^{1}$, Felicia Peterson Cavalher ${ }^{1}$, Jacqueline Montalvão \\ Araujo ${ }^{1}$, Caroline Petersen da Costa Ferreira ${ }^{2}$, Henrique Mateus ${ }^{3}$, Jacqueline Arantes Giannini Perlingeiro ${ }^{4}$, \\ José Cesar Assef ${ }^{5}$
}

\section{Resumo}

Introdução: Hemorragia digestiva alta (HDA) é um diagnostico comum no Pronto Socorro com diferentes etiologias. HDA é uma complicação rara de colelitíase, assim com sindrome de mirizzi. Apresentamos um caso de HDA por erosão de arteria gastroduodenal associada à Sindrome de Mirizzi. Relato de Caso: Paciente masculino 41 anos, imunossuprimido, admitido no pronto socorro com quadro clínico de hematêmese e instabilidade hemodinâmica. Após reposição volêmica e melhora hemodinâmica, foi submetido à endoscopia digestiva alta (EDA), onde foi evidenciado sangramento vultoso e em jato. Paciente apresentou piora hemodinâmica súbita, sendo a EDA suspensa e realizada laparotomia exploradora. No intraoperatório foi diagnosticada Síndrome de Mirizzi tipo Va, com fistula completa com duodeno, fístula incompleta com ducto hepatico comum e erosão da artéria Gastroduodenal com sangramento ativo. Foi realizada gastrectomia parcial e sutura da via biliar com drenagem à Kehr. Paciente evoluiu com choque séptico e foi a óbito dois dias depois. Conclusão: Sangramento digestivo alto secundário à Sindrome de Mirizzi é uma manifestação clinica bastante rara.

1. Graduado em Medicina pela Faculdade de Ciências Médicas da Santa Casa de São Paulo

2. Residente da Irmandade da Santa Casa de Misericórida de São Paulo. Departamento de Cirurgia

3. Médico Assistente da Irmandade da Santa Casa de Misericórida de São Paulo. Serviço de Emergência

4. Professora Assistente da Faculdade de Ciências Médicas da Santa Casa de São Paulo - Departamento de Cirurgia

5. Professor Adjunto da Faculdade de Ciências Médicas da Santa Casa de São Paulo - Departamento de Cirurgia / Chefe do Serviço de Emergência da Irmandade da Santa Casa de Misericórida de São Paulo

Trabalho realizado: Faculdade de Ciências Médicas da Santa Casa de São Paulo - Departamento de Cirurgia

Endereço ara correspondência: Maria Carolina Galli Mortati. Rua Afonso Celso, 694 - Vila Mariana -04119-060 - São PauloSP - Brasil. E-mail: carolmortati@gmail.com
Descritores: Síndrome de Mirizzi, Colelitíase, Colecistite

\begin{abstract}
Background: Upper Gastrointestinal Bleeding (UGB) is a common diagnosis in the Emergency Room with different etiologies. It is a rare complication of cholelithiasis, as well as mirizzi syndrome. We present a case of (UGB) due to erosion of the gastroduodenal artery associated with Mirizzi Syndrome. Case Report: Male, 41 years old, admitted to the emergency with upper gastrointestinal bleeding and hemodynamic instability. After hemodynamic stabilization, he went to endoscopy procedure but he developed a suddenly worsening with active bleeding coming from duodenum. The next step was an exploratory laparotomy. In the procedure he was diagnosed with Mirizzi syndrome type $\mathrm{Va}$, complete fistula with duodenum, incomplete fistula with common hepatic duct, and erosive lesion in gastroduodenal artery with active bleeding. Partial gastrectomy and bile duct suturing were performed with Kehr drainage. Patient developed septic shock and died two days later. Conclusion: Upper digestive bleeding secondary to Mirizzi Syndrome is a very rare clinical manifestation but it should be rememberd an upper gastrointestinal bleeding plus abdominal pain.
\end{abstract}

Keywords: Mirizzi syndrome, Cholelithiasis, Cholecystitis

\section{Introdução}

Hemorragia Digestiva Alta (HDA) é uma condição comum, com uma incidência de 40-150 casos por 100.000 habitantes, responsável por 1 a $2 \%$ das internações hospitalares de urgência no Brasil e tem mortalidade em torno de $10 \%$. HDA em $85 \%$ é de etiologia não varicosa e suas principais causas incluem: úlcera péptica em $28 \%-59 \%$, má formação arterio-venosa em 1\%-47\%; Síndrome de Mallory-Weiss em $4 \%-7 \%$ e tumores em $2 \%-4 \%$. Em geral, o tratamento das HDA's é clínico endoscópico, não necessitando abordagem cirúrgica ${ }^{(1)}$. 
A Síndrome de Mirizzi é uma complicação da colelitíase e consiste na presença de um cálculo no ducto cístico ou na ampola da vesícula biliar, pressionando o ducto colédoco e gerando obstrução do fluxo biliar devido a uma obstrução mecânica, seja diretamente pelo cálculo ou por conta de uma reação inflamatória secundária $^{(2-3)}$.

Os sintomas observados nessa síndrome são bastante inespecíficos, destacando-se dor abdominal em cólica em hipocôndrio direito e icterícia obstrutiva, que pode ser contínua ou intermitente. Outros sintomas menos prevalentes incluem náuseas, vômitos, febre, anorexia e colúria, sendo a hemorragia digestiva alta, uma manifestação bastante rara ${ }^{(4-6)}$.

Neste trabalho, relatamos um caso raro de um paciente com HDA consequente à erosão da parede da artéria grastroduodenal por Síndrome de Mirizzi com fístula colecistobiliar e colecistoduodenal.

\section{Relato de Caso}

Paciente do sexo masculino, 41 anos, procurou Serviço de Emergência com hematêmese em grande quantidade há 3 horas, dor abdominal em cólica há 7 dias da entrada, uso de anti-inflamatórios não esteroidais (AINES) nesse período e terapêutica anti-retroviral (TARV) desde 2011.

À admissão, paciente encontrava-se discretamente descorado, anictérico, instável hemodinamicamente, com pressão arterial (PA) de $90 \times 50 \mathrm{mmHg}$, taquicárdico com frequência cardíaca (FC) de $120 \mathrm{bpm}$, com pulsos periféricos finos, distensão abdominal, sem dor, apenas com desconforto à palpação profunda em epigástrio e hipocôndrio direito, sem evidência de melena no toque retal. Os exames laboratoriais estão representados na tabela 1 . Após reposição volêmica com $1000 \mathrm{ml}$ de cristaloide, ele apresentou normalização hemodinâmica e foi encaminhado para endoscopia digestiva alta (EDA) com a hipótese diagnóstica principal de úlcera péptica secundária ao uso de AINES.

Durante o procedimento endoscópico, foi identificada grande quantidade de sangue na câmara gástrica, presença de um bulbo duodenal distorcido, de difícil visualização. Foi observado sangramento vultoso, em jato, não sendo possível identificar sua origem. $\mathrm{O}$ paciente apresentou piora hemodinâmica, com PA inaudível, FC de 190 bpm, sendo a EDA suspensa e paciente encaminhado para a sala cirúrgica e submetido à laparotomia exploradora. No intra-operatório havia grande quantidade de sangue e coágulos oriundos de um bloqueio inflamatório em hipocôndrio direito. Após liberação do bloqueio foi identificado sangramento ativo oriundo da artéria gastroduodenal erodida, colecistite aguda calculosa com fístula colecisto-duodenal completa com abertura de mais $75 \%$ da circunferência do duodeno (Figura 1), além de fístula incompleta entre a vesícula e a parede anterior da via biliar (colédoco e hepático comum com $2 \mathrm{~cm}$ de extensão). Foi realizada ligadura da artéria gastroduodenal com controle do sangramento, colecistectomia à Torek, gastrectomia parcial, com gastroenteroanastomose à Billroth II e aproximação das bordas e sutura da parede anterior da via biliar (após realização de colangiografia para identificação das estruturas lesadas), com drenagem à Kehr (Figura 2). Durante a cirurgia recebeu 10 concentrados de hemácias, 5 plasmas frescos congelados e 7 UI de plaquetas e encontrava-se com hemoglobina de 9,2 ao final do procedimento. Paciente evoluiu instável apesar de altas doses de droga vasoativa, com choque séptico refratário e foi a óbito dois dias depois.

Tabela 1

Exames Laboratorias apresentados na Admissão

\begin{tabular}{lcc} 
Exames Laboratorias & $\begin{array}{c}\text { Valor na } \\
\text { Admissão }\end{array}$ & $\begin{array}{c}\text { Valor de referência em } \\
\text { homens }\end{array}$ \\
Hemoglobina & 10,0 & $13,5-17,5$ \\
\hline Leucograma & $\begin{array}{c}21.000 \\
10 \% \text { de } \\
\text { Bastões }\end{array}$ & $\begin{array}{c}5.000-10.000 / \mathrm{mm}^{3} \\
0-10 \%\end{array}$ \\
\hline Fosfatase Alcalina & 739 & $40-129 \mathrm{U} / \mathrm{L}$ \\
\hline Gama Glutamil & 1165 & $12-73 \mathrm{U} / \mathrm{L}$ \\
Transferase & 2,3 & $0.20-1.2 \mathrm{mg} / \mathrm{dL}$ \\
\hline Bilirrubina &
\end{tabular}

Fonte: Lee GR. Hemolytic disorders: general considerations. In: Lee GR, Foerster J, Lukens J, Paraskevas F, Greer JP, Rodgers GM, editors. Wintrobe's clinical hematology. $10^{\text {th }}$ ed. Philadelphia: Lippincott Williams \& Wilkins; 1998. v.1, p.1112-4 ${ }^{(7)}$

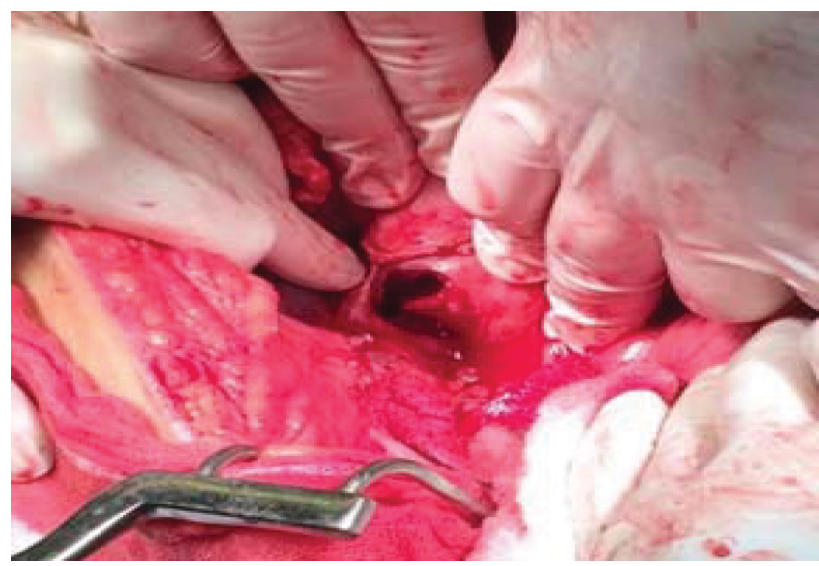

Figura 1 - Abertura do bulbo duodenal de aproximadamente $75 \%$ da circunferência do órgão

\section{Discussão}

Desde o início do século 49 discute-se a relação entre obstrução das vias biliares e a presença de cál- 


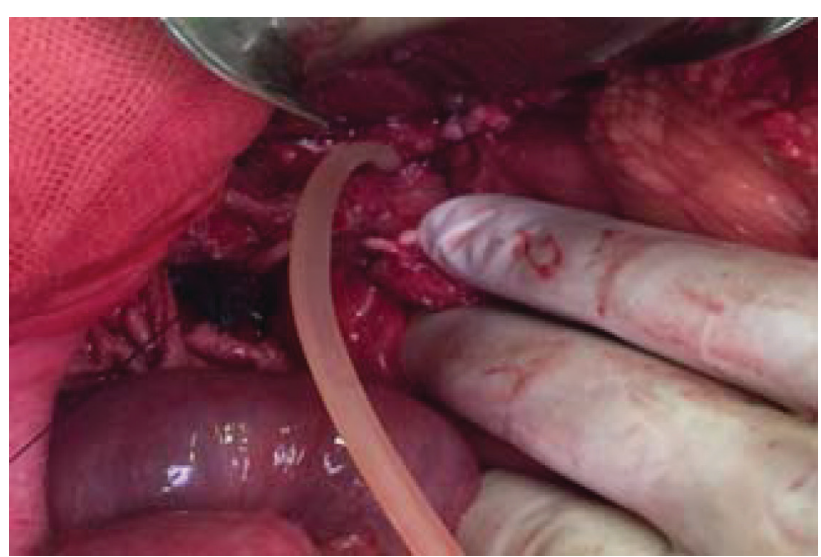

Figura 2 - Sutura do ducto hepático comum com colédoco utilizando dreno de Kehr como molde.

culos na vesicular biliar, porém apenas em 1948 tal condição clínica foi devidamente esclarecida por Pablo Mirizzi, sendo então batizada com seu sobrenome ${ }^{(8)}$. Assim, a Síndrome de Mirizzi pode ser explicada pela presença de um cálculo no ducto cístico ou na ampola da vesícula biliar, pressionando ducto colédoco. Como consequência, temos uma obstrução do fluxo biliar devido a uma obstrução mecânica, seja diretamente pelo cálculo ou por conta de uma reação inflamatória secundária ${ }^{(9)}$.

Segundo a classificação atual da Síndrome de Mirizzi, elaborada por Csendes et al $\left(1989^{(10)} ; 2007^{(11)}\right)$ e aprimorada por Beltran et al (2008) $)^{(12)}$, o tipo I consiste na compressão externa do ducto colédoco decorrente de um cálculo impactado no colo da vesícula biliar ou no ducto cístico, sendo o tipo de maior incidência. O tipo II corresponde à presença de uma fístula colecistobiliar (colecistohepática ou colecistocoledociana) decorrente da erosão da parede anterior ou lateral do ducto hepático comum ou do ducto cístico comum, respectivamente, sendo que a fístula acomete até um terço da circunferência do ducto biliar comum. O tipo III consiste na presença de uma fístula colecistobiliar com a erosão da parede do ducto biliar comum envolvendo até dois terços de sua circunferência. $\mathrm{O}$ tipo IV corresponde à presença de uma fístula colecistobiliar ocasionando a destruição completa de toda a parede do ducto biliar comum. Finalmente, o tipo V consiste em qualquer um dos tipos citados anteriormente associado a uma fístula colecistoentérica, tanto na ausência de íleo biliar (tipo Va) quanto em sua vigência (tipo $\mathrm{Vb}$ ) ${ }^{(10-12)}$. A Síndrome de Mirizzi, a fístula colecistocoledociana e íleo biliar são complicações raras da colelitíase. As duas primeiras complicações são manifestações do mesmo processo, que tem início com a impactação do cálculo no colo da vesícula biliar, resultando em obstrução do ducto hepático comum e, assim, icterícia. Os cálculos podem provocar a erosão do ducto biliar comum e adentrar seu interior, levando à fistulização. Já o íleo biliar consiste na obstrução do intestino delgado por um ou mais cálculos que migraram através de uma fístula colecistoentérica ${ }^{(8)}$. Existem alguns sinais e sintomas que podem ser sugestivos do diagnóstico de Síndrome de Mirizzi, como dor abdominal, icterícia e alterações séricas relacionadas à função hepática, porém nenhum destes é patognomônico da doença. Existem relatos na literatura de incidências menores de $66 \%$ de dor abdominal e icterícia em pacientes que foram posteriormente diagnosticados com Síndrome de Mirizzi ${ }^{(8-9)}$. A frequência desta síndrome é baixa, variando de $0,18 \%$ - 5,3\% na literatura, o que dificulta ainda mais o seu diagnóstico ${ }^{(4,8-10,12-15)}$. Assim, a apresentação de sinais e sintomas inespecíficos e com baixa frequência impede que o diagnóstico exclusivamente clínico pré-operatório seja confiável ${ }^{(9)}$. Apesar de alguns autores citarem métodos diagnósticos como $\mathrm{CPRE}^{(4,8-9,16)}$, na maioria das vezes o diagnóstico é intra-operatório.

O tratamento da Síndrome de Mirizzi é cirúrgico ${ }^{(4,8-9)}$, sendo baseado em três princípios básicos: a remoção do cálculo que levou à obstrução e da vesícula biliar; a restauração da integridade das vias biliares; e o fechamento da fístula colecistoentérica nos casos de Síndrome de Mirizzi tipo $\mathrm{V}^{(8)}$. O caso descrito neste artigo diferencia-se dos casos mais comuns de Síndrome de Mirizzi por sua apresentação clínica, pois, diferentemente da literatura, o sintoma que trouxe o paciente a emergência foi a hemorragia digestiva alta (HDA) e não dor abdominal ou icterícia, sintomas mais típicos da doença ${ }^{(4,9)}$. São relatados alguns outros casos de hemorragia digestiva alta como complicação de colecistite aguda, dentre elas, uma pseudo sindrome de Mirizzi por obstrução da via biliar por coágulo secundário a lesão de Dieulafoy na vesícula, cerca de trinta e cinco casos de sangramento por pseudoaneurismas rotos de arteria cística e um caso de sangramento secundário a fístula entre artéria hepática direita e ducto hepático comum após CPRE com colocação de prótese por síndrome de Mirizzi. Todavia, não há relatos de síndrome de Mirizzi grau Va manifestada por hemorragia digestiva alta secundária a rotura de arteria gastoduodenal, justificando a importância desse trabalho.

\section{Referências}

1. Gralnek IM, Dumoceau JM, Kuipers EJ, Lanas A, Sanders, DS, Kurien $\mathrm{M}$ et al. Diagnosis and management of nonvariceal upper gastrointestinal hemorrhage: European Society of Gastrointestinal Endoscopy (ESGE) Guideline. Endoscopy. 2015; 47:a1-a46.

2. Mirizzi PL. Syndrome del conducto hepatico. J Int Chir. 1948; 8:731-77.

3. Mirizzi PL. Hepatic duct syndrome. Cir Cir 1964; 32:641-64

4. Abou-Saif A, Al-Kawas FH. Complications of gallstone disease: 
Mirizzi syndrome, cholecystocholedochal fistula, and gallstone ileus. Am J Gastroenterol. 2002; 97(2):249-54.

5. Curet MJ, Rosendale DE, Congilosi S. Mirizzi syndrome in a Native American population. Am J Surg. 1994; 168(6):616-21.

6. Baer HU, Matthews JB, Schweizer WP, Gertsch P, Blumgart LH. Management of the Mirizzi syndrome and the surgical implications of cholecystcholedochal fistula. Br J Surg. 1990; 77(7):743-5.

7. Lee GR. Hemolytic disorders: general considerations. In: Lee GR, Foerster J, Lukens J, Paraskevas F, Greer JP, Rodgers GM, editors. Wintrobe's clinical hematology. $10^{\text {th }}$ ed. Philadelphia: Lippincott Williams \& Wilkins; 1998. v.1, p.1112-4.

8. Erben Y, Benavente-Chenhalls LA, Donohue JM, Que FG, Kendrick ML, Reid-Lombardo KM, et al. Diagnosis and treatment of Mirizzi syndrome: 23-year mayo clinic experience. J Am Coll Surg. 2011; 213(1):114-21.

9. Lampropoulos P, Paschalidis N, Marinis A, Rizos S. Mirizzi syndrome type Va: A rare coexistence of double cholecystobiliary and cholecysto-enteric fistulae. World J Radiol. 2010; 2(10):410-3.

10. Csendes A, Díaz JC, Burdiles P, Maluenda F, Nava O. Mirizzi syndrome and cholecystobiliary fistula: a unifying classification. Br J Surg. 1989; 76(11):1139-43.
11. Csendes A, Muñoz C, Alban M. Sindrome de Mirizzi - fistula colecistobiliar, una nueva clasificacion. Rev Chil Cir. 2007; 59(suppl):63-4.

12. Beltran MA, Csendes A, Cruces KS. The relationship of Mirizzi syndrome and cholecystoenteric fistula: validation of a modified classification. World J Surg. 2008; 32(10):2237-43.

13. Yetişir F, Şarer AE, Acar HZ, Parlak O, Basaran B, Yazıcıoğlu O. Laparoscopic resection of cholecystocolic fistula and subtotal cholecystectomy by tri-staple in a type V Mirizzi syndrome. Case Reports Hepatol. 2016; 2016:6434507.

14. Bower TC, Nagorney DM. Mirizzi syndrome. HPB Surg. 1988; 1(1):67-74; discussion:75-6.

15. Mishra MC, Vashishtha S, Tandon R. Biliobiliary fistula: preoperative diagnosis and management implications. Surgery 1990; 108(5):835-9.

16. Zaliekas J, Munson JL. Complications of gallstones: the mirizzi syndrome, gallstone ileus, gallstone pancreatitis, complications of "lost" gallstones. Surg Clin North Am. 2008; 88(6):1345-68.

Trabalho recebido: $17 / 07 / 2017$

Trabalho aprovado: 06/02/2018 